\title{
Tear trough deformity: different types of anatomy and treatment options
}

\author{
Jindou Jiang ${ }^{1}$, Xuekun Wang ${ }^{2}$, Rongrong Chen ${ }^{1}$, Xueying Xia ${ }^{1}$, Sai Sun ${ }^{1}$, Kuikui Hu${ }^{1}$
}

'Department of Aesthetic Surgery, Guangdong Woman and Children Hospital, Guangzhou Medical University, Guangzhou, China 2Department of Microbiology and Immunology, School of Medicine, Jinan University, Guangzhou, China

Adv Dermatol Allergol 2016; XXXIII (4): 303-308

DOI: 10.5114/ada.2016.61607

\begin{abstract}
Aim: To explore the efficacy of tear trough deformity treatment with the use of hyaluronic acid gel or autologous fat for soft tissue augmentation and fat repositioning via arcus marginalis release.

Material and methods: Seventy-eight patients with the tear trough were divided into three groups. Class I has tear trough without bulging orbital fat or excess of the lower eyelid skin. Class II is associated with mild to moderate orbital fat bulging, without excess of the lower eyelid skin. Class III is associated with severe orbital fat bulging and excess of the lower eyelid skin. Class I or II was treated using hyaluronic acid gel or autologous fat injections. Class III was treated with fat repositioning via arcus marginalis release. The patients with a deep nasojugal groove of class III were treated with injecting autologous fat into the tear trough during fat repositioning lower blepharoplasty as a way of supplementing the volume added by the repositioned fat.

Results: Seventy-eight patients with tear trough deformity were confirmed from photographs taken before and after surgery. There were some complications, but all had complete resolution.

Conclusions: Patients with mild to moderate peri-orbital volume loss without severe orbital fat bulging may be good candidates for hyaluronic acid filler or fat grafting alone. However, patients with more pronounced deformities, severe orbital fat bulging and excess of the lower eyelid skin are often better served by fat repositioning via arcus marginalis release and fat grafting.
\end{abstract}

Key words: tear trough deformities, fat grafting, hyaluronic acid, blepharoplasty.

\section{Introduction}

The tear trough is a concave deformity of the orbital fat that is noticeable as a result of inherited anatomical differences and aging. Natural facial rejuvenation has been achieved with the use of hyaluronic acid gel or autologous fat for soft tissue augmentation and fat repositioning via arcus marginalis release. The tear trough is a 2 or $3 \mathrm{~cm}$ depression, inferior to the pseudo herniated orbital fat of the lower eyelid [1-3]. It is characterized by a sunken appearance of the lower eyelid that results in the casting of a dark shadow over the nasal lower eyelid giving the patient a fatigued appearance $[4,5]$. The tear trough deformities can be a challenging area in facial rejuvenation. Understanding the different types of anatomy will provide an objective means of evaluating the deformity and aid the surgeon in choosing appropriate treat- ment options for correcting the tear trough deformity. Treatment options for the tear trough deformities include filler injection, fat grafting, lower blepharoplasty with fat repositioning or combining fat grafting in the tear trough.

\section{Aim}

In this article, we discuss the anatomy of the tear trough region and describe the most common surgical and nonsurgical treatment options.

\section{Material and methods}

A retrospective clinical study was conducted between May 2009 and September 2014 at the Guangdong Women and Children's Hospital. Seventy-eight consecutive patients (69 women and 9 men) with tear trough deformi-

Address for correspondence: Kuikui Hu, Department of Aesthetic Surgery, Guangdong Woman and Children Hospital, Guangyuanxilu No. 13, 510010 Guangzhou, China, phone: 15602332608, e-mail: huha4201587@163.com Received: 7.09.2015, accepted: 22.09.2015. 
ties were included in the study. All patients who agreed to participate signed consent form approved by the Human Subjects Review Board at the Guangdong Woman and Children's Hospital. The patients were between 25 and 62 years old (mean: $38.26 \pm 10.0$ years). The tear trough anatomy and aging were assessed using details of the physical examination and preoperative photographs. The following 3 variables were used: (1) volume loss, (2) orbital fat herniation, and (3) excess of the lower eyelid skin. Clinically, the tear trough pattern can be categorized into three classes. Class I group has tear trough without bulging orbital fat or excess of the lower eyelid skin. This group includes only tear trough without bulging orbital fat or excess of the lower eyelid skin, mild periorbital volume loss; flatness of mid-face.

Class II tear trough is associated with mild to moderate orbital fat bulging, without excess of the lower eyelid skin. This group includes mild to moderate periorbital volume loss; flatness of midface. Class III has significant periorbital volume loss; patients exhibit severe orbital fat bulging and excess of the lower eyelid skin. Patients present with a full depression circumferentially along the orbital rim medially to laterally.

Ten cases in our series were classified as class I, eighteen cases as class II and fifty cases as class III. Patients of class I or class II were treated using hyaluronic acid gel (18 cases) or autologous fat injections (10 cases). The patients who wish a simple, safe, and reversible nonsurgical procedure received hyaluronic acid gel filler. The hyaluronic acid (HA) gel filler Matrifill (EME China) was used in the injections. The injections were given using a 27-gauge needle. The filler was introduced by a serial technique with the needle advanced at a 90 angle through the skin, dermis, and orbicularis muscle until the needle tip reached the bone through one injection point. The injection was given in a retrograde fashion as the needle was slowly withdrawn. These were given just under the orbital rim, creating three column-shaped hyaluronic acid deposits deep in the orbicularis oculi muscle from $0.2 \mathrm{~mm}$ to $0.5 \mathrm{~mm}$. Treated areas were gently massaged immediately after the injection. Approximately $0.2-0.5 \mathrm{ml}$ was injected at a time per side. This technique created a deep scaffolding that could fill the orbital hollow $[6,7]$.

The patients who wish a long-lasting effect received fat grafting. Fat injections began with careful preoperative marking. We carefully defined the location of the tear trough, lid-cheek junction, and malar mound (if present) in each patient. Fat was harvested with a $20 \mathrm{ml}$ syringe under low suction pressure from the thigh area. The harvested fat was transferred into a stainless steel mesh strainer, washed in saline, and transferred back into the $1 \mathrm{ml}$ syringe via a connector. Using a blunt $0.9 \mathrm{~mm}$ and $1.2 \mathrm{~mm}$ cannula, multiple criss-crossing tunnels were created along multiple tissue planes and small amounts of fat were deposited deep to the orbicularis muscle along the tear trough as well as within the malar fat pad. If the nee- dle was placed too superficially, visible lumps of fat would will form in the future. Though the specific fat volume injected into each region was not specifically documented in the operative reports, the amount of fat injected into the tear trough unit generally totaled 0.5 to $2 \mathrm{ml}$ of fat and 2 to $5 \mathrm{ml}$ of fat into the malar region on each side [8].

Class III was treated with fat repositioning via arcus marginalis release (release of the orbicularis oculi muscle origin) (50 cases). Evaluating the patient in an animated state was vital for understanding how tissue needs to be rearranged. In an upright position, with the patient in neutral gaze, we marked out and then document each of the three fat compartments. We carefully defined the location of the tear trough, lid-cheek junction in each patient. A high subciliary incision was made through the skin at the lateral extent of the skin markings, and the skin flap was dissected by 4 to $5 \mathrm{~mm}$, after which the skin-muscle flap was elevated. Once the dissection reached the orbital rim, the orbicularis-retaining ligament at the orbital rim was dissected and elevated on the attached to the underlying zygoma caudal to the arcus marginalis periosteum. Dissection was carried out medially and inferiorly over the inferior orbital rim in the preperiosteal plane for a distance of about $10 \mathrm{~mm}$. The septa dividing the lower orbital fat into compartments were divided, allowing the fat to herniate across the entire infraorbital rim. The fat was carefully teased inferiorly and fixed to the periosteum with 5/0 Vicryl interrupted sutures. Lateral canthopexy or canthoplasty was simultaneously performed for elderly patients with weak lower lid tension. The skin was trimmed by 2 to $5 \mathrm{~mm}$, depending on the case, and then sutured [9]. The patients with a deep nasojugal groove of class III were treated with injecting autologous fat into the tear trough during fat repositioning lower blepharoplasty as a way of supplementing the volume added by the repositioned fat (11 cases).

\section{Results}

In the 78 patients, 67 patients were available for 3 months to 5 years' follow-up with 27 patients in class I or class II and 40 patients in class III. Improvements of the tear trough deformity were confirmed from photographs taken before and after surgery. Improvement in the tear trough deformity was achieved in all cases and all the patients were satisfied with the results. The level of postoperative patient satisfaction was assessed by interview and rated as follows: very satisfied, satisfied, acceptable, or not acceptable [10]. In class I or class II, 18 patients were treated using hyaluronic acid gel and 16 cases were available for follow-up (Figure 1 ). They show 6 months' to 12 months' maintaining time. Twelve patients rated themselves as very satisfied and 4 patients as satisfied. Two patients noticed not only volumetric correction but also improvement of their dark circle. No patients rated their level of satisfaction as acceptable or 
not acceptable. Two cases complained about lump after treatment. Hyaluronidase was found to resolve the lump effectively and rapidly after a single injection.

In class I or class II, 10 patients were treated with autologous fat injections and 9 cases were followed up. Three patients rated themselves as very satisfied, $4 \mathrm{pa}-$ tients as satisfied and 2 patients as acceptable. No patients rated their level of satisfaction as not acceptable. Complications included some degree of bruising (1/9), erythema (3/9), local swelling (9/9) and uneven after healing (2/9). It should be noted that swelling in the lower eyelid is expected for 5 or 14 days. In 1 case, long-lasting swelling was related to bruising and did not resolve until the bruise disappeared. Six patients underwent a second maintenance treatment and 2 patients underwent a third maintenance treatment. One case accepted the injection of hyaluronic acid gel because of fearing of surgery recovery time in the second treatment.

In class III, 40 patients were available for follow-up (Figures 2 and 3). Twenty patients rated themselves as very satisfied, 12 patients as satisfied and 8 patients as acceptable. No patient rated their level of satisfaction as not acceptable. There were two slight under-corrections. There was 1 patient older than 60 years who developed symptomatic lower eyelid malposition and required lateral canthopexies unilaterally and had complete resolution of her problem.

\section{Discussion}

The tear trough is a concave deformity rostral to the orbital fat that is noticeable as a result of inherited ana-
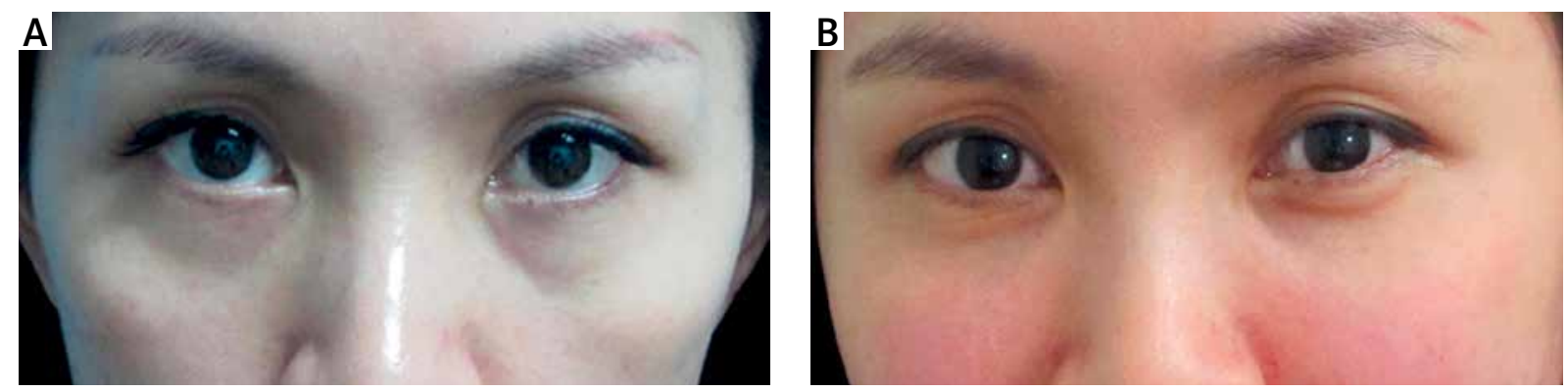

Figure 1. A - A 31-year-old woman in class I tear trough deformity before treatment. B - Marked improvement after injection of $0.4 \mathrm{ml}$ of hyaluronic acid filler per side
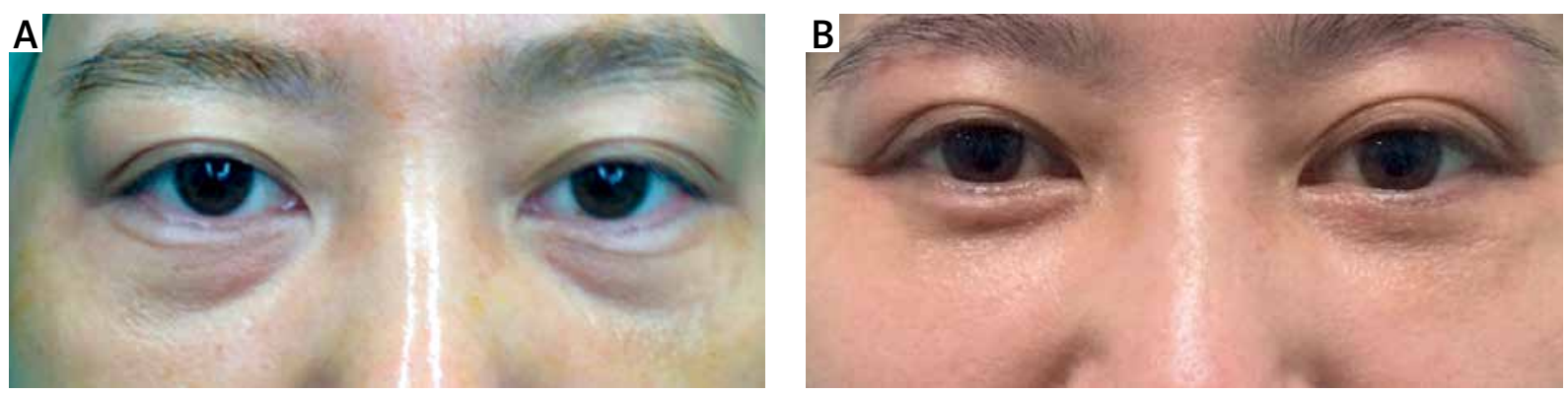

Figure 2. A - A 38-year-old woman in class III tear trough deformity before treatment. B - After treatment with fat repositioning lower blepharoplasty
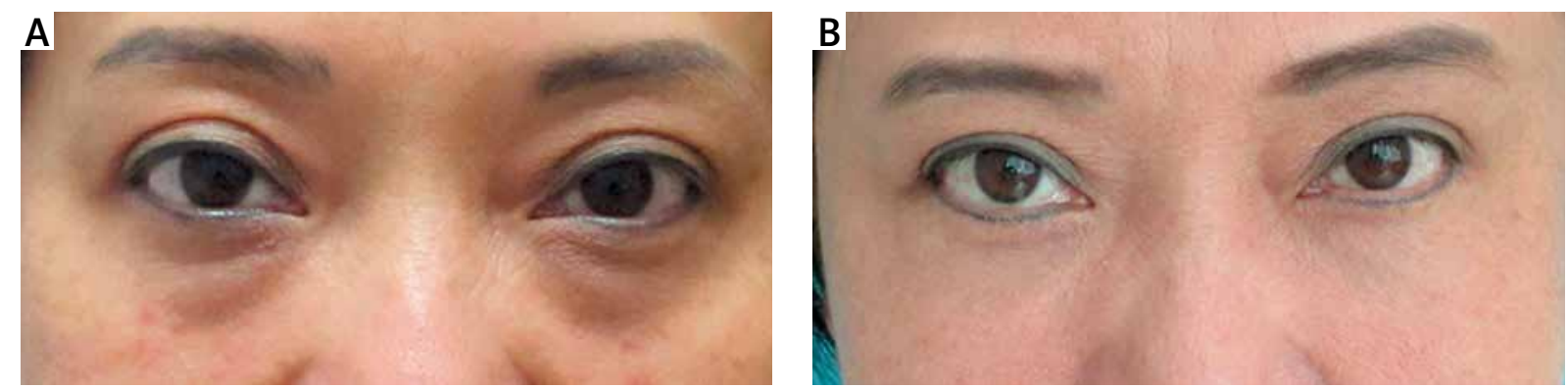

Figure 3. A - A 55-year-old woman in class III tear trough deformity before treatment. B - After treatment with injecting autologous fat into the tear trough during fat repositioning lower blepharoplasty 
tomical differences and aging. It may occur alone being a separate entity or in conjunction with lower eyelid bags [11]. There has been some debate regarding the contributing anatomy of tear trough deformity and the appropriate treatment options. In this article, we discuss the anatomy of the tear trough region and describe the most common surgical and nonsurgical treatment options.

\section{Anatomy}

Numerous theories have been proposed and previously described to explain the orbitomalar sulcus [12-15]. Various anatomic factors were related to tear trough deformities. Several reports have described and illustrated the tear trough deformity as a triangular defect bordered by the orbital portion of the orbicularis oculi (superiorly), the levator labii superioris (laterally), and the levator labii superioris alaeque nasi muscle (medially) $[4,16]$. However, results of more recent investigations have contradicted past anatomic descriptions. The position of the tear trough is most accurately described to be within the boundary of the orbicularis muscle $[12,17]$. Normal changes in the insertion of the orbicularis muscle, from medial to lateral, have permitted a better understanding of the anatomy and treatment [18]. As a result, the term "tear trough deformity" should be applied to the medial periorbital hollow extending obliquely from the medial canthus to the mid-pupillary line. There is absence of fat tissue from the central and medial fat pads subjacent to the orbicularis oculi muscle in the area below the groove. Volume bone loss of orbital rim seems to predominate in development of nasojugal groove and there is advanced volume loss in the central cheek, the medial cheek, and the malar eminence with aging. The integrity of the septum diminishes with advancing age such that orbital fat bulging leads to the appearance of bags or fullness in the lower eyelid. The tear trough deformity associated with aging has been explained by gravitational descent, such as laxity of the supporting ligaments and descent of the mid-face $[19,20]$. The orbicularis retaining ligament creates a V-shaped deformity that correlated with the lid-cheek junction. One of the reasons that aging adds to the deformity of tear trough is attributed to the loose orbicularis retaining ligament [21]. Lambros defined the tear trough as the junction of the thin, pigmented lower lid skin with the thicker cheek skin at the medial canthus to the mid-pupillary line. He found the lid-cheek junction to be stable over time and said that its perceived descent was due to age-related tissue volume changes and not actual movement [22]. The tear trough is often related to underlying bony structure and particularly associated with age-related maxillary hypoplasia [23]. Lower eyelid skin also progressively loses its elasticity and thickness with aging. Other changes in skin such as hyper-pigmentation, and actinic changes also play a role in the tear trough.

\section{Treatment options}

Aging is a dynamic process rather than a linear process and involves interactions between changes in various anatomic structures. Hereditary anatomic variations in these structures and the changes typically observed with aging must be considered in optimizing periocular treatment outcomes. Identifying anatomic problems involved with the tear trough could be able to choose customized surgical plan for each patient. By observing the groove formation mechanism and external performance, tear trough was divided into three groups. These differences are thought to have originated from inner anatomical relationships. Therefore, the goals of rejuvenating tear trough deformities surgery are different among the 3 groups. Volumetric enhancement of the infraorbital rim area or, alternatively, of the deep medial cheek, results in greater improvement of tear trough deformity. Class I and class II were treated using hyaluronic acid gel or autologous fat injection, class III was treated with fat repositioning via arcus marginalis release (release of the orbicularis oculi muscle origin). The patients with a deep nasojugal groove of class III were treated with injecting autologous fat into the tear trough during fat repositioning lower blepharoplasty as a way of supplementing the volume added by the repositioned fat.

Hyaluronic acid fillers are the most commonly used fillers worldwide [24-27]. The method is particularly appealing because it is perceived to be a simple, safe, and reversible nonsurgical procedure. Our study indicates that hyaluronic acid is well adapted for tear trough treatment, given the high satisfaction rate. Of course, there are some disadvantages, e.g. the treatment is not permanent. The superficial method can easily produce skin irregularities. In patients with large fat pads, only moderate improvement is possible with filler treatment, so they are not ideal candidates. Patients with excess skin on lower eyelids would also not experience the benefit that could be obtained with surgery. Some patients with lower eyelid hyperpigmentation (dark circles) may experience improvement in their appearance after the treatment, but this cannot be guaranteed.

The effect of hyaluronic acid fillers is temporary. Transplanted fat yields more-lasting volume enhancement than hyaluronic acid fillers, because some of the injected autologous cells remain viable after injection. In addition, unlike synthetic fillers, autologous fat has the ability to change in structure with the patient's physiologic changes and adverse reactions are extremely uncommon. Fat transplantation has local improvements in skin quality [28-31]. It was not possible to accurately quantify how much fat was injected into the tear trough and malar region, but as a general principle in this technique, 0.5 to $1.5 \mathrm{ml}$ are injected into the tear trough region and 2 to $3 \mathrm{ml}$ in the malar region. Furthermore, it is difficult to assess what percentage of fat remains viable due to its physical properties [32, 33]. 
The use of autologous fat may offer some advantages when compared with open surgical volume enhancement, it is less invasive than surgery and can be repeated if desired by patients. Furthermore, the volume of injection is customizable for each patient and the source is also readily available with minimal concern of infection. However, the skin and soft tissue overlying the tear trough area are very thin and may result in palpable and visible contour abnormalities if the fat is not precisely placed. Imprecise placement of the fat, especially when injected in isolation into the tear trough region, can also lead to a sausage-shaped deformity. The other disadvantages of fat injection include the need to harvest the fat, unpredictable absorption of the fat, and the need for multiple sessions.

Blepharoplasty was revolutionized by Loeb, who proposed periorbital fat preservation and used this fat to smooth the nasojugal groove [34-36]. Early descriptions of lower lid blepharoplasty focused on removal of tissue, particularly skin and subcutaneous fat [37]. Removal of fat can increase an already hallow appearance, particularly of the superior sulcus, without really rejuvenating the periorbital area. The facial bones in the lower periorbital region also lose volume, contributing to focal deflation and loss of eyelid support in the inferior orbital rim area. Fat repositioning is a good option for patients with adequate orbital fat and a significant tear trough depression [38-40]. However, the tear trough deformity could not be completely resolved with this technique for patients with a deep nasojugal groove. Optimal effacement is limited by the amount of fat available for transposition, the viability of the transposted fat, and a steep learning curve. The authors began to inject autologous fat into this space during fat repositioning lower blepharoplasty as a way of supplementing the volume added by the repositioned fat. In this way, they could more fully treat the tear trough depression than with fat repositioning alone with improved esthetic results. It was believed that there is a synergistic effect between these 2 procedures, which enables successful rejuvenation of the tear trough and the eyelid-cheek junction.

\section{Conclusions}

Lower eyelid rejuvenation with treatment of the tear trough deformity can be a challenging endeavor. Understanding of the complex factors that contribute to teartrough deformity changes during the aging process may allow for adequate and customized surgery for each patient. Patients with mild to moderate periorbital volume loss without severe orbital fat bulging may be good candidates for HA filler or fat grafting alone. However, patients with more pronounced deformities, severe orbital fat herniation and excess of the lower eyelid skin are often better served by lower blepharoplasty with fat repositioning or combining fat grafting. Esthetically-pleasing results are possible through knowledge of the periorbital anatomy and proper treatment selection.

\section{Acknowledgments}

We thank all participants for very kind volunteering to take part in this study. We also thank Liyan Li and Bifang Zhang for valuable assistance.

\section{Conflict of interest}

The authors declare no conflict of interest.

\section{References}

1. Flowers RS. Tear trough implants for correction of tear trough deformity. Clin Plast Surg 1993; 20: 403-15.

2. Loeb R. Fat pad sliding and fat grafting for leveling lid depressions. Clin Plast Surg 1981; 8: 757-76.

3. Espinoza GM, Holds JB. Evaluation and treatment of the tear trough deformity in lower blepharoplasty. Semin Plast Surg 2007; 21: 57-64.

4. Hirmand H. Anatomy and nonsurgical correction of the tear trough deformity. Plast Reconstr Surg 2010; 125: 699-708.

5. Hirsch RJ, Carruthers JD, Carruthers A. Infraorbital hollow treatment by dermal fillers. Dermatol Surg 2007; 33: 1116-9.

6. Berros P, Lax L, Bétis F. Hyalurostructure treatment: superior clinical outcome through a new protocol-a 4-year comparative study of two methods for tear trough treatment. Plast Reconstr Surg 2013; 132: 924e-31e.

7. De Pasquale A, Russa G, Pulvirenti M, Di Rosa L. Hyaluronic acid filler injections for tear-trough deformity: injection technique and high-frequency ultrasound follow-up evaluation. Aesthetic Plast Surg 2013; 37: 587-91.

8. Willemsen JC, Mulder KM, Stevens HP. Lipofilling with minimal access cranial suspension lifting for enhanced rejuvenation. Aesthet Surg J 2011; 31: 759-69.

9. Hidalgo DA. An integrated approach to lower blepharoplasty. Plast Reconstr Surg 2011; 127: 386-95.

10. Liao SL, Wei YH. Fat repositioning via supraperiosteal dissection with internal fization for tear trough deformity in an Asian population. Graefes Arch Clin Exp Ophthalmol 2011; 249: 1735-41.

11. Berros P, Lax L, Bétis F. Hyalurostructure treatment: superior clinical outcome through a new protocol-a 4-year comparative study of two methods for tear trough treatment. Plast Reconstr Surg 2013; 132: 924e-31e.

12. Haddock NT, Saadeh PB, Boutros S, Thorne CH. The tear trough and lid/cheek junction: anatomy and implications for surgical correction. Plast Reconstr Surg 2009; 123: 1332-40.

13. Ghavami A, Pessa JE, Janis J, et al. The orbicularis retaining ligament of the medial orbit: closing the circle. Plast Reconstr Surg 2008; 121: 994-1001.

14. Rohrich RJ, Arbique GM, Wong C, et al. The anatomy of suborbicularis fat: implications for periorbital rejuvenation. Plast Reconstr Surg 2009; 124: 946-51.

15. Mendelson BC, Jacobson SR. Surgical anatomy of the midcheek: facial layers, spaces, and the midcheek segments. Clin Plast Surg 2008; 35: 395-404.

16. Codner MA, Wolfli JN, Anzarut A. Primary transcutaneous lower blepharoplasty with routine lateral canthal support: a comprehensive 10-year review. Plast Reconstr Surg 2008; 121: 241-50. 
17. Mendelson BC, Muzaffar AR, Adams WP. Surgical anatomy of the midcheek and malar mounds. Plast Reconstr Surg 2002; 110: 885-96.

18. Muzaffar AR, Mendelson BC, Adams WP. Surgical anatomy of the ligamentous attachments of the lower lid and lateral canthus. Plast Reconstr Surg 2002; 110: 873-84.

19. Shorr N, Hoenig JA, Goldberg RA, et al. Fat preservation to rejuvenate the lower eyelid. Arch Facial Plast Surg 1999; 1: 38-9.

20. LaFerriere KA, Kilpatrick JK. Transblepharoplasty: subperiosteal approach to rejuvenation of the aging midface. Facial Plast Surg 2003; 19: 157-70.

21. Yang N, Qiu W, Wang Z, et al. Anatomical studying of the tear trough area. Zhonghua Zheng Xing Wai Ke Za Zhi 2014; 30: 50-3.

22. Lambros V. Observation on periorbital and midface aging. Plast Reconstr Surg 2007; 120: 1367-76.

23. Goldberg RA, McCann JD, Fiaschetti D, Ben Simon GJ. What causes eyelid bags? Analysis of 114 consecutive patients. Plast Reconstr Surg 2005; 115: 1395-402.

24. Lambros VS. Hyaluronic acid injections for correction of the tear trough deformity. Plast Reconstr Surg 2007; 120 (6S): 74S-80S.

25. Goldberg RA, Lee S, Jayasundera T, et al. Treatment of lower eyelid retraction by expansion of the lower eyelid with hyaluronic acid gel. Ophthal Plast Reconstr Surg 2007; 23: 343-8.

26. Morley AM, Malhotra R. Use of hyaluronic acid filler for teartrough rejuvenation as an alternative to lower eyelid surgery. Ophthal Plast Reconstr Surg 2011; 27: 69-73.

27. Peckinpaugh JL, Reddy HS, Tower RN. Large particle hyaluronic acid gel for the treatment of lower eyelid retraction associated with radiation-induced lipoatrophy. Ophthal Plast Reconstr Surg 2010; 26: 377-9.

28. Coleman SR. Structural fat grafting: more than a permanent filler. Plast Reconstr Surg 2006; 118: 108S-20S.

29. Strem BM, Hicok KC, Zhu M, et al. Multipotential differentiation of adipose tissue-derived stem cells. Keio J Med 2005; 54: 132-41.

30. De Ugarte DA, Ashjian PH, Elbarbary A, et al. Future of fat as raw material for tissue regeneration. Ann Plast Surg 2003; 50: 215-9.

31. Tanzi MC, Fare S. Adipose tissue engineering: state of the art, recent advances and innovative approaches. Expert Rev Med Devices 2009; 6: 533-51.

32. Coleman SR. Facial recontouring with lipostructure. Clin Plast Surg 1997; 24: 347-67.

33. Coleman S. Periorbital rejuvenation. Aesthet Surg J 2001; 21: 337-43.

34. Loeb R. Aesthetic surgery of the eyelids. Springer-Verlag, New York 1989.

35. Loeb R. Naso-jugal groove leveling with fat tissue. Clin Plast Surg 1993; 20: 393-400.

36. Loeb R. Fat pad sliding and fat grafting for leveling lid depressions. Clin Plast Surg 1981; 8: 757-76.

37. Parkes M, Fein W. Further experience with the pinch technique for repair of eyelid deformities. Arch Ophthalmol 1976; 94: 1534-6.

38. Parkes M, Fein W, Brennan HG. Pinch technique for repair of cosmetic eyelid deformities. Arch Ophthalmol 1973; 89: 324-8.

39. Baker SR. Orbital fat preservation in lower-lid blepharoplasty. Arch Facial Plast Surg 1999; 1: 33-7.

40. Hamra ST. Arcus marginalis release and orbital fat preservation in midface rejuvenation. Plast Reconstr Surg 1995; 96: 354-62. 